\title{
z

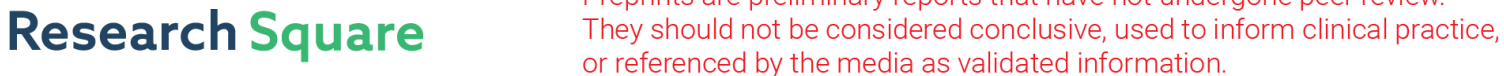

\section{Prevalence, Risk, and Outcome of Deep Vein Thrombosis in Acute Respiratory Distress Syndrome}

$\mathrm{Na}$ Cui

Beijing Chao-Yang Hospital: Beijing Chaoyang Hospital

Chunguo Jiang

Beijing Chao-Yang Hospital: Beijing Chaoyang Hospital

Hairong Chen

Shandong Qianfuo Mountain Hospital: Shandong Qianfoshan Hospital

Yuan Gao

Shandong Qianfuo Mountain Hospital: Shandong Qianfoshan Hospital

\section{Lifeng Yu}

Shandong Qianfuo Mountain Hospital: Shandong Qianfoshan Hospital

\section{Liming Zhang}

Beijing Chao-Yang Hospital: Beijing Chaoyang Hospital

Xiaokai Feng ( $\square$ fengxiaokai2020@163.com )

Beijing Chao-Yang Hospital: Beijing Chaoyang Hospital https://orcid.org/0000-0002-1521-9545

\section{Research}

Keywords: respiratory distress syndrome (ARDS), deep vein thrombosis (DVT), hypoxemia

Posted Date: May 21st, 2021

DOI: https://doi.org/10.21203/rs.3.rs-543193/v1

License: (a) (1) This work is licensed under a Creative Commons Attribution 4.0 International License. Read Full License 


\section{Abstract}

Background: Few data exist on deep vein thrombosis (DVT) in patients with acute respiratory distress syndrome (ARDS), a group of heterogeneous diseases characterized by acute hypoxemia.

Study Design and Methods: We retrospectively enrolled 225 adults with ARDS admitted to the Beijing Chao-Yang Hospital and the First Affiliated Hospital of Shandong First Medical University between 1 January 2015 and 30 June 2020. We analyzed clinical, laboratory, and echocardiography data for groups with and without DVT and for direct and indirect ARDS subgroups.

Results: Ninety (40.0\%) patients developed DVT. Compared with the non-DVT group, patients with DVT were older, had lower serum creatinine levels, lower partial pressure of arterial oxygen/fraction of inspired oxygen, higher serum procalcitonin levels, higher Padua prediction scores, and higher proportions of sedation and invasive mechanical ventilation (IMV). Multivariate analysis showed an association between age, serum creatinine level, IMV, and DVT in the ARDS cohort. The sensitivity and specificity of corresponding receiver operating characteristic curves were not inferior to those of the Padua prediction score and the Caprini score for screening for DVT in the three ARDS cohorts. Patients with DVT had a significantly lower survival rate than those without DVT in the overall ARDS cohort and in the groups with direct and indirect ARDS.

Conclusions: The prevalence of DVT is high in patients with ARDS. The risk factors for DVT are age, serum creatinine level, and IMV, which predict DVT and are superior to the Padua prediction score and the Caprini score. DVT is associated with decreased survival in patients with ARDS.

\section{Introduction}

Deep vein thrombosis (DVT) and pulmonary embolism (PE), collectively referred to as venous thromboembolism (VTE), constitute a major global burden of disease [1]. Some studies demonstrated an increased risk of VTE in patients in the intensive care unit (ICU) [2-4]. Patients with acute respiratory distress syndrome (ARDS) are at high risk for DVT because they are susceptible both to general risk factors for VTE and to those specific to the critically ill, such as advanced age, sedation, immobilization, insertion of a central venous catheter, and mechanical ventilation (MV), combined with a severe inflammatory response and hypercoagulable states [2-6]. ARDS remains under-recognized clinically; however, therapies are limited, complications are frequent, and mortality remains significantly high [711]. Patients with ARDS are a heterogeneous group with significant variability in clinical presentation and outcomes. One approach to reducing these heterogeneities is to subclassify patients with ARDS as having direct (pulmonary) or indirect (extrapulmonary) ARDS based on variabilities in the pathological, radiological, and respiratory mechanical responses to different management strategies [12-19].

The incidence of DVT in patients with direct and indirect ARDS has not been investigated. 
We performed a multi-institutional study to identify the prevalence, risk factors, and prognosis of DVT and to determine whether the predictors of DVT differed between direct and indirect ARDS in a cohort of patients identified with ARDS.

\section{Methods}

\section{Study Design and Population}

We retrospectively enrolled adult patients ( $\geq 18$ years old) with ARDS (according to the Berlin definition) [8] who were admitted to the Department of Pulmonary and Critical Care Medicine, Beijing Chao-Yang Hospital and the Intensive Care Unit, the First Affiliated Hospital of Shandong First Medical University, from 1 January 2015 to 30 June 2020. Patients with ARDS were classified as having direct ARDS or indirect ARDS based on the underlying risk factors for ARDS recorded by study personnel. Patients with pneumonia and aspiration as risk factors and those with pulmonary sepsis were assigned to the direct ARDS group, whereas those with pancreatitis or non-pulmonary sepsis were assigned to the indirect ARDS group. Some known diseases such as active malignant tumor, lower limb fracture, hip and knee arthroplasty, major trauma and many more that could cause DVT were excluded. The first ultrasound examination should be performed within 1-3 days after the diagnosis of ARDS, and then the ultrasound examination should be reexamined again according to the patient's condition. After intensive treatment, if the patient remained unstable because of conditions such as unexplained hypoxemia or cardiac insufficiency, he or she should be reexamined by ultrasound. If there was more than one ultrasound scan for a single patient, all the results were recorded. Patients were divided into a DVT and a non-DVT group according to the results of the venous ultrasound scans. The flow chart is shown in Fig. 1 (A, B).

The study was approved by the ethics committees of the Beijing Chao-Yang Hospital (2020-ke-429) and the First Affiliated Hospital of Shandong First Medical University (S003) and was conducted in accordance with the 1964 Helsinki Declaration and its later amendments or comparable ethical standards.

\section{Clinical Data}

We analyzed the medical records of the enrolled patients. Data, which included demographic information, clinical history, vital signs, laboratory findings, treatments, complications, and outcomes of the patients during hospitalization, were collected and analyzed. We analyzed the survival rates of all patients within 28 days after a diagnosis of ARDS. For patients discharged within 28 days, we followed up by telephone concerning their survival status after discharge.

\section{Ultrasound Study}

Bedside ultrasound examinations were performed using a portable color ultrasound scanner (CX50, Philips Medical Systems, the Netherlands, equipped with an L12-3/S5-1 probe). The lower extremity venous compression ultrasound and echocardiographic data were obtained from the institution's Picture Archiving and Communication System. The levels of DVT included the bilateral common femoral, deep 
and superficial femoral veins, the popliteal veins, and the anterior tibial, posterior tibial, peroneal, and calf muscle veins. Left ventricular and right ventricular function parameters were captured. The presence of pulmonary artery hypertension was evaluated by adding a tricuspid regurgitation pressure gradient to the estimated right atrial pressure [20].

\section{Definitions}

ARDS was defined according to the Berlin definition [8]. Sepsis was defined according to the Third International Consensus Definitions for Sepsis and Septic Shock [21]. A distal thrombosis was defined as a thrombosis in the veins of the calf muscle or in at least 1 branch of the 3 pairs of deep calf veins (anterior tibial vein, posterior tibial vein, or peroneal vein); a proximal thrombosis was defined as a thrombosis in the popliteal vein or above. The Caprini score was defined according to the updated Caprini Risk Assessment Model (2013 Version) [22]. The Padua prediction score was defined according to the Barbar model [23]. We applied the Acute Physiology and Chronic Health Evaluation (APACHE) $\triangle$ score and the Sequential Organ Failure Assessment (SOFA) score to assess the severity of disease [21, 24, 25].

\section{Statistical Analyses}

Categorical variables were described as number and percentage (\%) and continuous variables, as mean, standard deviation, median, and interquartile range. The Shapiro-Wilk test was used to verify normality. Differences between the DVT and the non-DVT groups were assessed by a two-sample $t$ test for normally distributed continuous variables, the Mann-Whitney $U$ test for non-normally distributed continuous variables, and the $\chi 2$ or Fisher exact test for categorical variables. To determine risk factors for DVT, multivariable logistic regression analysis was performed on the direct and the indirect ARDS subgroups. The adjusted odds ratio (OR) with $95 \%$ confidence intervals $(\mathrm{Cl})$ was reported. To further evaluate the observed differences in risk factors for DVT between direct and indirect ARDS, we utilized interaction terms between ARDS type and each risk factor. A receiver operating characteristic (ROC) analysis was performed to calculate the sensitivity and specificity of risk factors for screening for DVT. The area under receiver operating curves (ROC-AUCs) for different risk factors were compared using the method of DeLong et al. Survival curves were plotted using the Kaplan-Meier method and compared between patients with or without DVT using the log-rank test. All statistical analyses were performed using the Statistical Analysis System, version 9.4 (SAS Institute, Cary, NC, USA). All tests were two-tailed; $P<0.05$ was considered statistically significant.

\section{Results}

A total of 225 patients with ARDS were enrolled in this study; 111 patients were considered to belong in the direct ARDS group and 114 patients, in the indirect ARDS group. The flow chart is shown in Fig. 1 (A, B).

Table 1 shows the demographic and clinical characteristics of patients with direct and indirect ARDS. Compared with the direct ARDS group, patients with indirect ARDS had higher APACHE II scores $(P=$ 0.010), higher SOFA scores $(P<0.001)$, higher white blood cell counts $(P<0.001)$, a higher neutrophil 
count $(P<0.001)$, higher levels of procalcitonin $(P=0.008)$, higher levels of serum creatinine $(P=0.019)$, and higher levels of D-dimer $(P<0.001)$. There were more men in the direct ARDS group $(P<0.001)$. Patients with direct ARDS had lower $\mathrm{PaO}_{2} / \mathrm{FiO}_{2}$ than those with indirect ARDS $(P<0.001)$. 
Table 1

Demographic and Clinical Characteristics of Patients with Direct and Indirect ARDS

\begin{tabular}{|c|c|c|c|c|}
\hline Characteristic & $\begin{array}{l}\text { Total } \\
(\mathrm{N}=225)\end{array}$ & $\begin{array}{l}\text { Direct ARDS } \\
(n=111)\end{array}$ & $\begin{array}{l}\text { Indirect } \\
\text { ARDS }(n=114)\end{array}$ & $\begin{array}{l}P \\
\text { value }\end{array}$ \\
\hline Age (years) & $66 \pm 17$ & $64 \pm 15$ & $67 \pm 18$ & 0.192 \\
\hline Male, n (\%) & $144(64.0)$ & $84(75.7)$ & $60(52.6)$ & $\begin{array}{l}< \\
0.001\end{array}$ \\
\hline BMI & $24.1(21.6,26.8)$ & $24.0(21.0,26.2)$ & $24.4(21.9,27.0)$ & 0.274 \\
\hline Bedridden time (days) & $8(4,15)$ & $9(5,15)$ & $8(4,16)$ & 0.400 \\
\hline APACHE II score & $23(19,28)$ & $22(17,27)$ & $25(19,31)$ & 0.010 \\
\hline SOFA score & $8(5,10)$ & $6(4,6)$ & $9(6,11)$ & $<.001$ \\
\hline \multicolumn{5}{|l|}{ Laboratory data } \\
\hline White blood cells $(\times 109 / L)$ & $16.9(12.0,21.5)$ & $14.3(10.1,20.3)$ & $18.2(14.4,23.5)$ & $<.001$ \\
\hline Neutrophils (×109/L) & $14.5(10.4,19.7)$ & $12.8(9.0,17.7)$ & $16.1(12.6,20.8)$ & $<.001$ \\
\hline Platelets (×109/L) & $\begin{array}{l}183.0(101.0 \\
253.5)\end{array}$ & $\begin{array}{l}190.0(120.0 \\
270.0)\end{array}$ & $\begin{array}{l}167.0(73.8, \\
238.0)\end{array}$ & 0.094 \\
\hline C-reactive protein(mg/L) & $\begin{array}{l}120.0(89.4 \\
120.0)\end{array}$ & $\begin{array}{l}120.0(85.0 \\
120.0)\end{array}$ & $\begin{array}{l}120.0(92.2 \\
120.0)\end{array}$ & 0.391 \\
\hline Procalcitonin (ng/mL) & $5.6(2.0,16.2)$ & $4.1(1.5,13.5)$ & $7.3(3.0,21.2)$ & 0.008 \\
\hline Serum creatinine $(\mu \mathrm{mol} / \mathrm{L})$ & $\begin{array}{l}116.7(66.6 \\
209.5)\end{array}$ & $90.5(65.0,193.0)$ & $\begin{array}{l}136.9(67.9 \\
248.8)\end{array}$ & 0.019 \\
\hline D-dimer $(\mu \mathrm{g} / \mathrm{ml})$ & $1.9(0.9,3.8)$ & $1.3(0.6,2.5)$ & $2.4(1.3,5.3)$ & $\begin{array}{l}< \\
0.001\end{array}$ \\
\hline
\end{tabular}

Data are mean $\pm \mathrm{SD}$, median (IQR) or $\mathrm{n}(\%)$. $P$ values comparing Direct and Indirect ARDS groups were from a two-sample $t$-test, Mann- Whitney $U$ test, or $\chi^{2}$ test. $P<0.05$ was considered statistically significant.

$\chi^{2}$ test comparing Direct and Indirect ARDS groups.

$\chi^{2}$ test comparing all subcategories.

Abbreviations: APACHE, Acute Physiology and Chronic Health Evaluation; ARDS, acute respiratory disease syndrome; $\mathrm{BMI}$, body mass index; DVT, deep venous thrombosis; $\mathrm{FiO}_{2}$, fraction of inspired oxygen; ICU, intensive care unit; IQR, interquartile range; mild, $200 \mathrm{mmHg} \square \mathrm{PaO}_{2} / \mathrm{FiO}_{2} \leq 300 \mathrm{mmHg}$; moderate, $100 \mathrm{mmHg} \square \mathrm{PaO}_{2} / \mathrm{FiO}_{2} \leq 200 \mathrm{mmHg} ; \mathrm{PaO}_{2}$, partial pressure of arterial oxygen; SD, standard deviation; severe, $\mathrm{PaO}_{2} / \mathrm{FiO}_{2} \leq 100 \mathrm{mmHg}$; SOFA, Sequential Organ Failure Assessment. 


\begin{tabular}{|c|c|c|c|c|}
\hline Characteristic & $\begin{array}{l}\text { Total } \\
(\mathrm{N}=225)\end{array}$ & $\begin{array}{l}\text { Direct ARDS } \\
(n=111)\end{array}$ & $\begin{array}{l}\text { Indirect } \\
\text { ARDS }(n=114)\end{array}$ & $\begin{array}{l}P \\
\text { value }\end{array}$ \\
\hline $\mathrm{PaO}_{2} / \mathrm{FiO}_{2}$ & $158(103,199)$ & $136(80,186)$ & $170(130,208)$ & $<.001$ \\
\hline Mild, n (\%) & $54(24.0)$ & $22(19.8)$ & $32(28.1)$ & $<.001$ \\
\hline Moderate, n (\%) & $116(51.6)$ & $49(44.1)$ & $67(58.8)$ & \\
\hline Severe, n (\%) & $55(24.4)$ & $40(36.0)$ & $15(13.2)$ & \\
\hline DVT, n (\%) & $90(40.0 \%)$ & $44(39.6)$ & $46(40.4)$ & 0.913 \\
\hline ICU length of stay(days) & $11(6,24)$ & $13(7,25)$ & $10(5,24)$ & 0.103 \\
\hline $\begin{array}{l}\text { Hospital length of } \\
\text { stay(days) }\end{array}$ & $19(12,32)$ & $17(10,29)$ & $22(13,34)$ & 0.055 \\
\hline Mortality, n (\%) & $77(34.2)$ & $36(32.4)$ & $41(36.0)$ & 0.577 \\
\hline \multicolumn{5}{|c|}{$\begin{array}{l}\text { Data are mean } \pm \mathrm{SD} \text {, median }(\mathrm{IQR}) \text { or } \mathrm{n}(\%) . P \text { values comparing Direct and Indirect ARDS groups were } \\
\text { from a two-sample } t \text { test, Mann- Whitney } U \text { test, or } \chi^{2} \text { test. } P<0.05 \text { was considered statistically } \\
\text { significant. }\end{array}$} \\
\hline \multicolumn{5}{|c|}{$\chi^{2}$ test comparing Direct and Indirect ARDS groups. } \\
\hline \multicolumn{5}{|c|}{$\chi^{2}$ test comparing all subcategories. } \\
\hline \multicolumn{5}{|c|}{$\begin{array}{l}\text { Abbreviations: APACHE, Acute Physiology and Chronic Health Evaluation; } \mathrm{ARDS} \text {, acute respiratory } \\
\text { disease syndrome; } \mathrm{BMI} \text {, body mass index; } \mathrm{DVT} \text {, deep venous thrombosis; } \mathrm{FiO}_{2} \text {, fraction of inspired } \\
\text { oxygen; ICU, intensive care unit; IQR, interquartile range; mild, } 200 \mathrm{mmHg} \mathrm{PaO}_{2} / \mathrm{FiO}_{2} \leq 300 \mathrm{mmHg} \text {; } \\
\text { moderate, } 100 \mathrm{mmHg} \mathrm{maaO} / \mathrm{FiO}_{2} \leq 200 \mathrm{mmHg} ; \mathrm{PaO}_{2} \text {, partial pressure of arterial oxygen; } \mathrm{SD} \text {, standard } \\
\text { deviation; severe, } \mathrm{PaO}_{2} / \mathrm{FiO}_{2} \leq 100 \mathrm{mmHg} \text {; SOFA, Sequential Organ Failure Assessment. }\end{array}$} \\
\hline
\end{tabular}

Some patients underwent more than one ultrasound scan. The median number of ultrasound examinations was 1 (range, $1-5$ ). Of the 225 patients, 90 (40.0\%) developed DVT, including 7 with proximal DVT and 83 with distal DVT, 73 of whom had muscular calf vein thrombosis only. Five patients were clinically suspected of having PE; three were further confirmed by computed tomography pulmonary angiography (CTPA) examination. There was no difference in the prevalence of DVT in patients with direct and indirect ARDS (39.6\% [44/111] vs 40.4\% [46/114], respectively; $P=0.913)$.

Table 2 shows the demographic and clinical characteristics of patients with ARDS. Compared with the non-DVT group, patients with DVT were older $(P<0.001)$ and had lower levels of serum creatinine $(P=$ 0.007), lower levels of partial pressure of arterial oxygen/fraction of inspired oxygen $\left(\mathrm{PaO}_{2} / \mathrm{FiO}_{2} ; P=\right.$ 0.002), higher levels of serum procalcitonin (PCT; $P<0.001)$, higher Padua prediction scores $(P=0.023)$, and a higher proportion of patients given sedative therapy $(P=0.001)$ and invasive mechanical 
ventilation (IMV; $P<0.001)$. Patients with DVT had more deaths within 28 days after ARDS than those without DVT $(P<0.001)$. There were no differences in comorbidities $(P>0.05$ for all; data are not shown) between the DVT and the non-DVT groups. All patients were bedridden for more than 3 days with no difference between the DVT and the non-DVT groups $(P=0.216)$. For the $135(60.0 \%)$ patients who received VTE prophylaxis, the incidence of DVT was 37.0\% (50/135); however, for the patients who did not receive VTE prophylaxis, it was $44.4 \%$ (40/90), and there was no significant difference between the two groups $(P=0.271)$. For the $108(48.0 \%)$ patients who received low molecular weight heparin, the incidence of DVT was $36.1 \%$ (39/108), and for the 75 (33.5\%) patients who received combined treatment with low molecular weight heparin and physical prevention, it was $37.3 \%(28 / 75)$. There was no significant difference between the two groups $(P=0.866)$. Among the 90 patients who did not receive VTE prophylaxis, 32 had anticoagulant therapy contraindications, such as stress ulcers and gastrointestinal bleeding (17 patients), platelet counts less than $50 \times 10^{9} / \mathrm{L}$ (13 patients), and haemoptysis (2 patients). The remaining $58(25.8 \%)$ patients had no clear high-risk factors for bleeding but did not receive VTE prophylaxis due to the actual clinical situation at that time in this retrospective observational study. A total of 215 (95.6\%) patients received echocardiographic examinations, with 86 patients in the DVT group and 129 patients in the non-DVT group (Table 3). Compared with the non-DVT group, patients with DVT had a lower left ventricular end-systolic volume index $(P=0.041)$ and higher pulmonary artery systolic pressure $(P=0.007)$. 
Table 2

Demographic and Clinical Characteristics of DVT Vs Non-DVT Patients in Overall ARDS Cohort

\begin{tabular}{|c|c|c|c|c|}
\hline Characteristic & Total & DVT & Non-DVT & $\begin{array}{l}P \\
\text { value }\end{array}$ \\
\hline & $(N=225)$ & $(n=90)$ & $(n=135)$ & \\
\hline Age (years) & $66 \pm 17$ & $70 \pm 13$ & $63 \pm 18$ & $\begin{array}{l}< \\
0.001\end{array}$ \\
\hline Male, n (\%) & $144(64.0)$ & $52(57.8)$ & $92(68.1)$ & 0.112 \\
\hline BMI & $24.1(21.6,26.8)$ & $24.0(20.8,26.0)$ & $24.2(22.0,27.2)$ & 0.146 \\
\hline Bedridden time (days) & $8(4,15)$ & $10(5,18)$ & $7(4,15)$ & 0.216 \\
\hline Caprini score & $7(5,9)$ & $7(5,10)$ & $7(5,9)$ & 0.135 \\
\hline Padua prediction score & $6(5,6)$ & $6(5,8)$ & $5(5,6)$ & 0.023 \\
\hline APACHE II score & $23(19,28)$ & $24(20,28)$ & $23(18,29)$ & 0.596 \\
\hline SOFA score & $8(5,10)$ & $7(5,9)$ & $8(5,11)$ & 0.622 \\
\hline \multicolumn{5}{|l|}{ Laboratory data } \\
\hline White blood cells $(\times 109 / L)$ & $16.9(12.0,21.4)$ & $16.5(13.4,21.0)$ & $17.1(11.6,22.6)$ & 0.733 \\
\hline Neutrophils (×109/L) & $14.5(10.4,19.7)$ & $14.7(11.4,19.3)$ & $14.4(9.9,20.1)$ & 0.709 \\
\hline Platelets (×109/L) & $\begin{array}{l}183.0(101.0 \\
253.5)\end{array}$ & $\begin{array}{l}196.5(124.3 \\
263.3)\end{array}$ & $\begin{array}{l}172.0(84.0 \\
253.0)\end{array}$ & 0.141 \\
\hline C-reactive protein(mg/L) & $\begin{array}{l}120.0(89.4 \\
120.0)\end{array}$ & $\begin{array}{l}120.0(82.0 \\
120.0)\end{array}$ & $\begin{array}{l}120.0(92.5 \\
120.0)\end{array}$ & 0.471 \\
\hline Procalcitonin (ng/mL) & $5.6(2.0,16.2)$ & $12.7(3.4,21.5)$ & $3.5(1.5,10.3)$ & $\begin{array}{l}<.001 \\
0.001\end{array}$ \\
\hline
\end{tabular}

Data are presented as mean \pm SD, median (IQR), or $\mathrm{n}(\%)$. $P$ values comparing DVT and non-DVT groups were from a two-sample $t$-test, Mann-Whitney $U$ test, $\chi^{2}$ test, or Fisher exact test. $P<0.05$ was considered statistically significant.

$\chi^{2}$ test comparing DVT and non-DVT groups.

$\chi^{2}$ test comparing all subcategories.

Abbreviations: APACHE, Acute Physiology and Chronic Health Evaluation; ARDS, acute respiratory disease syndrome; BMI, body mass index; CRRT, continuous renal replacement therapy; CVC, central venous catheterization; DVT, deep venous thrombosis; $\mathrm{FiO}_{2}$, fraction of inspired oxygen; ICU, intensive care unit; IMV, invasive mechanical ventilation; IQR, interquartile range; LMWH, low molecular weight heparin; mild, $200 \mathrm{mmHg} \square \mathrm{PaO}_{2} / \mathrm{FiO}_{2} \leq 300 \mathrm{mmHg}$; moderate, $100 \mathrm{mmHg} \square \mathrm{PaO}_{2} / \mathrm{FiO}_{2} \leq 200 \mathrm{mmHg}$; $\mathrm{PaO}_{2}$, partial pressure of arterial oxygen; $\mathrm{SD}$, standard deviation; severe, $\mathrm{PaO}_{2} / \mathrm{FiO}_{2} \leq 100 \mathrm{mmHg}$; SOFA, Sequential Organ Failure Assessment; VTE, venous thromboembolism. 


\begin{tabular}{|lllll|}
\hline Characteristic & Total & DVT & Non-DVT & $\begin{array}{l}P \\
\text { value }\end{array}$ \\
\hline Serum creatinine $(\mu \mathrm{mol} / \mathrm{L})$ & $\begin{array}{l}116.7(66.6, \\
209.5)\end{array}$ & $\begin{array}{l}95.1(58.8, \\
165.5)\end{array}$ & $\begin{array}{l}125.6(70.6, \\
250.3)\end{array}$ & 0.007 \\
\hline D-dimer $(\mu \mathrm{g} / \mathrm{ml})$ & $1.9(0.9,3.8)$ & $2.1(0.9,5.0)$ & $1.8(0.9,3.3)$ & 0.070 \\
\hline $\mathrm{PaO}_{2} / \mathrm{FiO}_{2}$ & $158(103,199)$ & $137(87,179)$ & $172(116,209)$ & 0.002 \\
\hline Mild, $\mathrm{n}(\%)$ & $54(24.0)$ & $14(15.6)$ & $40(29.6)$ & 0.026 \\
\hline Moderate, $\mathrm{n}(\%)$ & $116(51.6)$ & $48(53.3)$ & $68(50.4)$ & \\
\hline Severe, $\mathrm{n}(\%)$ & $55(24.4)$ & $28(31.1)$ & $27(20.0)$ & \\
\hline Treatments & & & & \\
\hline Glucocorticoid therapy, $\mathrm{n}(\%)$ & $51(22.7)$ & $22(24.4)$ & $29(21.5)$ & 0.603 \\
\hline Immunoglobulin, $\mathrm{n}(\%)$ & $5(2.2)$ & $2(2.2)$ & $3(2.2)$ & 1.000 \\
\hline Sedative therapy, $\mathrm{n}(\%)$ & $96(42.7)$ & $50(55.6)$ & $46(34.1)$ & 0.001 \\
\hline $\begin{array}{l}\text { Vasoactive agent therapy, } \mathrm{n} \\
\text { (\%) }\end{array}$ & $55(24.4)$ & $25(27.8)$ & $30(22.2)$ & 0.342 \\
\hline CRRT, $\mathrm{n}(\%)$ & $33(14.7)$ & $10(11.1)$ & $23(17.0)$ & 0.218 \\
\hline CVC, $\mathrm{n}$ (\%) & $125(55.6)$ & $55(61.1)$ & $70(51.9)$ & 0.171 \\
\hline IMV, $\mathrm{n}$ (\%) & $122(54.2)$ & $66(73.3)$ & $56(41.5)$ & 0.001 \\
\hline Length of IMV (days) & $3(2,7)$ & $3(2,6)$ & $3(2,8)$ & 0.543 \\
\hline Length of IMV $\geq 3$ days, $\mathrm{n}(\%)$ & $80(65.6)$ & $43(65.2)$ & $37(66.1)$ & 0.915 \\
\hline VTE prophylaxis, $\mathrm{n}(\%)$ & $135(60.0)$ & $50(55.6)$ & $85(63.0)$ & 0.267 \\
\hline
\end{tabular}

Data are presented as mean $\pm \mathrm{SD}$, median (IQR), or $\mathrm{n}(\%)$. $P$ values comparing DVT and non-DVT groups were from a two-sample $t$-test, Mann-Whitney $U$ test, $\chi^{2}$ test, or Fisher exact test. $P<0.05$ was considered statistically significant.

$\chi^{2}$ test comparing DVT and non-DVT groups.

$\chi^{2}$ test comparing all subcategories.

Abbreviations: APACHE, Acute Physiology and Chronic Health Evaluation; ARDS, acute respiratory disease syndrome; BMI, body mass index; CRRT, continuous renal replacement therapy; CVC, central venous catheterization; DVT, deep venous thrombosis; $\mathrm{FiO}_{2}$, fraction of inspired oxygen; ICU, intensive care unit; IMV, invasive mechanical ventilation; IQR, interquartile range; $L M W H$, low molecular weight heparin; mild, $200 \mathrm{mmHg} \square \mathrm{PaO}_{2} / \mathrm{FiO}_{2} \leq 300 \mathrm{mmHg}$; moderate, $100 \mathrm{mmHg} \square \mathrm{PaO} 2 / \mathrm{FiO}_{2} \leq 200 \mathrm{mmHg}$; $\mathrm{PaO}_{2}$, partial pressure of arterial oxygen; $\mathrm{SD}$, standard deviation; severe, $\mathrm{PaO}_{2} / \mathrm{FiO}_{2} \leq 100 \mathrm{mmHg}$; SOFA, Sequential Organ Failure Assessment; VTE, venous thromboembolism. 


\begin{tabular}{|c|c|c|c|c|}
\hline Characteristic & Total & DVT & Non-DVT & $\begin{array}{l}P \\
\text { value }\end{array}$ \\
\hline LMWH, n (\%) & $108(48.0)$ & $39(43.3)$ & $69(51.1)$ & 0.253 \\
\hline $\begin{array}{l}\text { LMWH + physical prophylaxis, } \\
\text { n (\%) }\end{array}$ & $75(33.5)$ & $29(32.6)$ & $46(34.1)$ & 0.817 \\
\hline $\begin{array}{l}\text { Physical prophylaxis only, } \mathrm{n} \\
(\%)\end{array}$ & $23(10.3)$ & $9(10.1)$ & $14(10.4)$ & 0.950 \\
\hline ICU length of stay(days) & $11(6,24)$ & $12(5,24)$ & $11(6,26)$ & 0.563 \\
\hline Hospital length of stay(days) & $19(12,32)$ & $20(11,32)$ & $19(12,31)$ & 0.816 \\
\hline Mortality, n (\%) & $77(34.2)$ & $45(50.0)$ & $32(23.7)$ & $<0.001$ \\
\hline \multicolumn{5}{|c|}{$\begin{array}{l}\text { Data are presented as mean } \pm \mathrm{SD} \text {, median }(\mathrm{IQR}) \text {, or } \mathrm{n}(\%) . P \text { values comparing DVT and non-DVT } \\
\text { groups were from a two-sample } t \text {-test, Mann-Whitney } U \text { test, } \chi^{2} \text { test, or Fisher exact test. } P<0.05 \text { was } \\
\text { considered statistically significant. }\end{array}$} \\
\hline \multicolumn{5}{|c|}{$\chi^{2}$ test comparing DVT and non-DVT groups. } \\
\hline \multicolumn{5}{|c|}{$\chi^{2}$ test comparing all subcategories. } \\
\hline \multicolumn{5}{|c|}{ 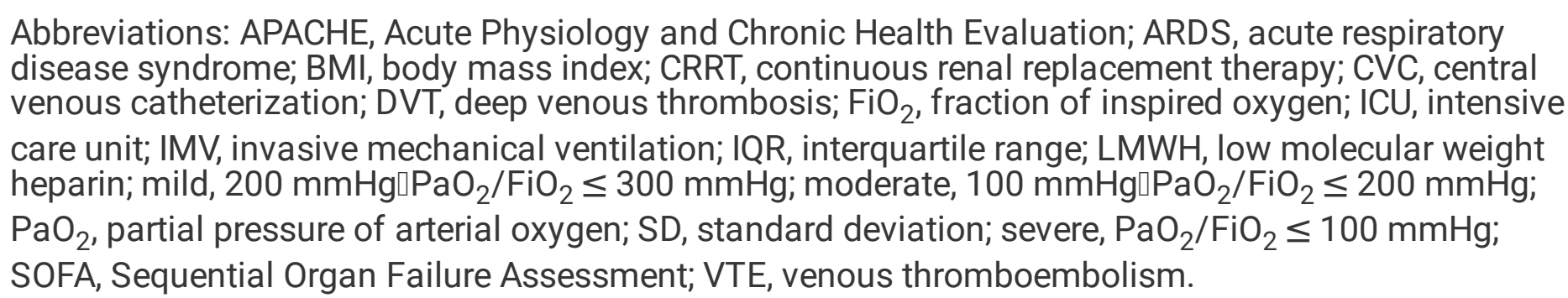 } \\
\hline
\end{tabular}


Table 3

Echocardiographic Findings of DVT Vs Non-DVT Patients in Overall ARDS Cohort

\begin{tabular}{|c|c|c|c|c|}
\hline Variables & Total & DVT & Non-DVT & $P$ value \\
\hline LA diameter (mm) & $48(43,53)$ & $47(42,53)$ & $48(43,52)$ & 0.764 \\
\hline LVESVI (mL/m2) & $46(43,49)$ & $46(42,48)$ & $47(44,49)$ & 0.041 \\
\hline LVEDVI (mL/m2) & $29(27,32)$ & $29(26,31)$ & $30(27,32)$ & 0.107 \\
\hline Simpson biplane EF (\%) & $66(62,70)$ & $67(62,70)$ & $66(61,70)$ & 0.513 \\
\hline RA diameter (mm) & $45(41,48)$ & $45(41,48)$ & $44(40,48)$ & 0.236 \\
\hline RV diameter (mm) & $30(26,32)$ & $30(27,32)$ & $29(26,32)$ & 0.839 \\
\hline PA diameter (mm) & $23(21,25)$ & $23(22,25)$ & $23(21,25)$ & 0.933 \\
\hline PASP (mmHg) & $40(36,48)$ & $43(38,60)$ & $38(35,46)$ & 0.007 \\
\hline PAH, n (\%) & $55(25.6)$ & $25(29.1)$ & $30(23.3)$ & 0.338 \\
\hline Pericardial effusion & $12(5.6)$ & $4(4.7)$ & $8(6.2)$ & 0.628 \\
\hline Tricuspid regurgitation & $86(40.0)$ & $38(44.2)$ & 48 (37.2) & 0.306 \\
\hline \multicolumn{5}{|c|}{$\begin{array}{l}\text { Data are presented as median (IQR) or } \mathrm{n}(\%) . P \text { values comparing DVT and non-DVT were from Mann- } \\
\text { Whitney } U \text { test, or } \chi^{2} \text { test. } P<0.05 \text { was considered statistically significant. }\end{array}$} \\
\hline \multicolumn{5}{|c|}{$\begin{array}{l}\text { Abbreviations: ARDS, acute respiratory disease syndrome; DVT, deep vein thrombosis; EF, ejection } \\
\text { fraction; LA, left atrial; LVEDVI, left ventricular end-diastolic volume index; LVESVI, left ventricular end- } \\
\text { systolic volume index; PA, pulmonary artery; PAH, pulmonary artery hypertension; PASP, pulmonary } \\
\text { artery systolic pressure; RA, right atrial; RV, right ventricular. }\end{array}$} \\
\hline
\end{tabular}

Table 4 shows the clinical characteristics of patients with and without DVT in the direct and indirect ARDS cohorts. In the direct and indirect ARDS cohorts, patients with DVT were older, had a lower $\mathrm{PaO}_{2} / \mathrm{FiO}_{2}$, a higher level of $\mathrm{PCT}$, and a higher proportion who were given sedative therapy and IMV than patients without DVT $(P<0.05$ for all). Patients with DVT had lower serum creatinine levels $(P=0.003)$ in the direct ARDS cohort and higher Caprini scores $(P=0.021)$ and higher Padua prediction scores $(P=$ $0.008)$ in the indirect ARDS cohort. More patients with DVT died within 28 days after being diagnosed with ARDS than those without DVT in both groups ( $P=0.005$ and $P=0.003$, respectively). There were no differences in APACHE $₫$ scores and SOFA scores between patients with and without DVT regardless of ARDS subgroup. 
Table 4

Demographic

and Clinical

Characteristics

of DVT Vs

Non-DVT

Patients in

Direct and

Indirect ARDS

Cohorts

Page 13/30 


\begin{tabular}{|c|c|c|c|c|c|c|}
\hline & $\begin{array}{l}\text { DVT } \\
(n=44)\end{array}$ & $\begin{array}{l}\text { Non-DVT } \\
(n=67)\end{array}$ & $\begin{array}{l}\mathrm{P} \\
\text { Value }\end{array}$ & $\begin{array}{l}\text { DVT } \\
(n= \\
46)\end{array}$ & $\begin{array}{l}\text { Non- } \\
\text { DVT } \\
(n=68)\end{array}$ & $\begin{array}{l}P \\
\text { value }\end{array}$ \\
\hline Age (years) & $68 \pm 11$ & $62 \pm 17$ & 0.015 & $\begin{array}{l}72 \pm \\
15\end{array}$ & $64 \pm 19$ & 0.009 \\
\hline Male, n (\%) & $32(72.7)$ & $52(77.6)$ & 0.557 & $\begin{array}{l}20 \\
(43.5)\end{array}$ & $\begin{array}{l}40 \\
(58.8)\end{array}$ & 0.107 \\
\hline BMI & $\begin{array}{l}23.5 \\
(20.6 \\
25.2)\end{array}$ & $\begin{array}{l}24.2(22.2, \\
27.2)\end{array}$ & 0.076 & $\begin{array}{l}24.5 \\
(21.3 \\
26.8)\end{array}$ & $\begin{array}{l}24.0 \\
(22.0 \\
27.3)\end{array}$ & 0.716 \\
\hline Bedridden time (days) & $9(6,17)$ & $9(5,15)$ & 0.431 & $10(4,19)$ & $7(4,14)$ & 0.356 \\
\hline Caprini score & $7(5,8)$ & $7(5,9)$ & 0.891 & $9(6,11)$ & $7(5,10)$ & 0.021 \\
\hline Padua prediction score & $5(5,6)$ & $5(5,6)$ & 0.689 & $7(5,8)$ & $6(4,7)$ & 0.008 \\
\hline APACHE II score & $\begin{array}{l}22(17, \\
26)\end{array}$ & $21(16,27)$ & 0.568 & $\begin{array}{l}25(21, \\
30)\end{array}$ & $\begin{array}{l}25(19, \\
32)\end{array}$ & 0.913 \\
\hline SOFA score & $6(5,9)$ & $6(4,10)$ & 0.780 & $9(7,10)$ & $9(6,11)$ & 0.772 \\
\hline \multicolumn{7}{|l|}{ Laboratory data } \\
\hline White blood cells $(\times 109 / L)$ & $\begin{array}{l}14.5 \\
(10.0 \\
18.3)\end{array}$ & $\begin{array}{l}14.1(10.2, \\
20.7)\end{array}$ & 0.921 & $\begin{array}{l}18.4 \\
(15.0 \\
21.5)\end{array}$ & $\begin{array}{l}18.1 \\
(13.1 \\
24.2)\end{array}$ & 0.630 \\
\hline Neutrophils (×109/L) & $\begin{array}{l}12.8(9.1, \\
16.8)\end{array}$ & $\begin{array}{l}12.8(8.9 \\
18.0)\end{array}$ & 0.935 & $\begin{array}{l}16.1 \\
(13.3, \\
19.8)\end{array}$ & $\begin{array}{l}16.1 \\
(11.0 \\
21.8)\end{array}$ & 0.669 \\
\hline
\end{tabular}

Data are presented as mean $\pm S D$, median (IQR), or $n(\%)$. P values comparing DVT and non-DVT groups were from a two-sample t-test, Mann-Whitney $U$ test, $\chi^{2}$ test, or Fisher exact test. $P<0.05$ was considered statistically significant.

$\chi^{2}$ test comparing DVT and non-DVT groups.

$\chi^{2}$ test comparing all subcategories.

Abbreviations: APACHE, Acute Physiology and Chronic Health Evaluation; ARDS, acute respiratory disease syndrome; $\mathrm{BMI}$, body mass index; CRRT, continuous renal replacement therapy; CVC, central venous catheterization; DVT, deep venous thrombosis; $\mathrm{FiO}_{2}$, fraction of inspired oxygen; ICU, intensive care unit; IMV, invasive mechanical ventilation; IQR, interquartile range; $L M W H$, low molecular weight heparin; mild, $200 \mathrm{mmHg} \square \mathrm{PaO}_{2} / \mathrm{FiO}_{2} \leq 300 \mathrm{mmHg}$; moderate, $100 \mathrm{mmHg} \square \mathrm{PaO}{ }_{2} / \mathrm{FiO}_{2} \leq 200 \mathrm{mmHg}$; $\mathrm{PaO}_{2}$, partial pressure of arterial oxygen; $\mathrm{SD}$, standard deviation; severe, $\mathrm{PaO}_{2} / \mathrm{FiO}_{2} \leq 100 \mathrm{mmHg}$; SOFA, Sequential Organ Failure Assessment; VTE, venous thromboembolism. 


\begin{tabular}{|c|c|c|c|c|c|c|}
\hline & $\begin{array}{l}\text { DVT } \\
(n=44)\end{array}$ & $\begin{array}{l}\text { Non-DVT } \\
(n=67)\end{array}$ & $\begin{array}{l}\mathrm{P} \\
\text { Value }\end{array}$ & $\begin{array}{l}\text { DVT } \\
(n= \\
46)\end{array}$ & $\begin{array}{l}\text { Non- } \\
\text { DVT } \\
(n=68)\end{array}$ & $\begin{array}{l}\mathrm{P} \\
\text { value }\end{array}$ \\
\hline Platelets (×109/L) & $\begin{array}{l}193.5 \\
(155.3 \\
279.0)\end{array}$ & $\begin{array}{l}181.0 \\
(108.0 \\
257.0)\end{array}$ & 0.301 & $\begin{array}{l}202.0 \\
(84.3 \\
246.5)\end{array}$ & $\begin{array}{l}148.5 \\
(70.8, \\
234.0)\end{array}$ & 0.267 \\
\hline C-reactive protein $(\mathrm{mg} / \mathrm{L})$ & $\begin{array}{l}120.0 \\
(82, \\
120.0)\end{array}$ & $\begin{array}{l}120.0 \\
(89.0,120.0)\end{array}$ & 0.490 & $\begin{array}{l}120.0 \\
(86.8 \\
120.0)\end{array}$ & $\begin{array}{l}120.0 \\
(94.4 \\
120.0)\end{array}$ & 0.758 \\
\hline Procalcitonin (ng/mL) & $\begin{array}{l}8.3(2.6 \\
17.6)\end{array}$ & $\begin{array}{l}2.6(1.1 \\
9.6)\end{array}$ & 0.002 & $\begin{array}{l}15.0(4.9 \\
25.0)\end{array}$ & $\begin{array}{l}4.9(2.6, \\
13.5)\end{array}$ & 0.001 \\
\hline Serum creatinine $(\mu \mathrm{mol} / \mathrm{L})$ & $\begin{array}{l}77.3 \\
(56.2 \\
122.4)\end{array}$ & $\begin{array}{l}119.0 \\
(70.6 \\
225.1)\end{array}$ & 0.003 & $\begin{array}{l}135.5 \\
(67.3 \\
208.8)\end{array}$ & $\begin{array}{l}140.7 \\
(70.0 \\
275.7)\end{array}$ & 0.377 \\
\hline D-dimer $(\mu \mathrm{g} / \mathrm{ml})$ & $\begin{array}{l}1.8(0.7 \\
3.2)\end{array}$ & $\begin{array}{l}1.2(0.6 \\
2.1)\end{array}$ & 0.211 & $\begin{array}{l}3.2(1.6 \\
7.3)\end{array}$ & $\begin{array}{l}2.1(1.3, \\
4.7)\end{array}$ & 0.123 \\
\hline $\mathrm{PaO} 2 / \mathrm{FiO} 2$ & $\begin{array}{l}111 \text { (71, } \\
176)\end{array}$ & $\begin{array}{l}150(86, \\
203)\end{array}$ & 0.035 & $\begin{array}{l}152(117, \\
184)\end{array}$ & $\begin{array}{l}179 \\
(140, \\
217)\end{array}$ & 0.017 \\
\hline Mild, n (\%) & 5 (11.4) & $17(25.4)$ & 0.062 & $9(19.6)$ & $\begin{array}{l}23 \\
(33.8)\end{array}$ & 0.249 \\
\hline Moderate, n (\%) & $18(40.9)$ & $31(46.3)$ & & $30(65.2)$ & $\begin{array}{l}37 \\
(54.4)\end{array}$ & \\
\hline $\begin{array}{l}\text { Severe, } \mathrm{n}(\%) 21(47.7) 19 \\
(28.4) 7(15.2) 8(11.8)\end{array}$ & $21(47.7)$ & $19(28.4)$ & & $7(15.2)$ & $8(11.8)$ & \\
\hline
\end{tabular}

Data are presented as mean $\pm S D$, median (IQR), or $n(\%)$. $P$ values comparing DVT and non-DVT groups were from a two-sample t-test, Mann-Whitney $U$ test, $\chi^{2}$ test, or Fisher exact test. $P<0.05$ was considered statistically significant.

$\chi^{2}$ test comparing DVT and non-DVT groups.

$\chi^{2}$ test comparing all subcategories.

Abbreviations: APACHE, Acute Physiology and Chronic Health Evaluation; ARDS, acute respiratory disease syndrome; $\mathrm{BMI}$, body mass index; CRRT, continuous renal replacement therapy; CVC, central venous catheterization; DVT, deep venous thrombosis; $\mathrm{FiO}_{2}$, fraction of inspired oxygen; ICU, intensive care unit; IMV, invasive mechanical ventilation; IQR, interquartile range; LMWH, low molecular weight heparin; mild, $200 \mathrm{mmHg} \square \mathrm{PaO}_{2} / \mathrm{FiO}_{2} \leq 300 \mathrm{mmHg}$; moderate, $100 \mathrm{mmHg} \square \mathrm{PaO} 2 / \mathrm{FiO}_{2} \leq 200 \mathrm{mmHg}$; $\mathrm{PaO}_{2}$, partial pressure of arterial oxygen; $\mathrm{SD}$, standard deviation; severe, $\mathrm{PaO}_{2} / \mathrm{FiO}_{2} \leq 100 \mathrm{mmHg}$; SOFA, Sequential Organ Failure Assessment; VTE, venous thromboembolism. 


\begin{tabular}{|c|c|c|c|c|c|c|}
\hline & $\begin{array}{l}\text { DVT } \\
(n=44)\end{array}$ & $\begin{array}{l}\text { Non-DVT } \\
(n=67)\end{array}$ & $\begin{array}{l}\mathrm{P} \\
\text { Value }\end{array}$ & $\begin{array}{l}\text { DVT } \\
(n= \\
46)\end{array}$ & $\begin{array}{l}\text { Non- } \\
\text { DVT } \\
(n=68)\end{array}$ & $\begin{array}{l}\mathrm{P} \\
\text { value }\end{array}$ \\
\hline Glucocorticoid therapy, n (\%) & $16(36.4)$ & $15(22.4)$ & 0.108 & $6(13.0)$ & $\begin{array}{l}14 \\
(20.6)\end{array}$ & 0.299 \\
\hline Immunoglobulin, n (\%) & $1(2.3)$ & $2(3.0)$ & 1.000 & $1(2.2)$ & $1(1.5)$ & 1.000 \\
\hline Sedative therapy, n (\%) & $25(56.8)$ & $25(37.3)$ & 0.043 & $25(54.3)$ & $\begin{array}{l}21 \\
(30.9)\end{array}$ & 0.012 \\
\hline $\begin{array}{l}\text { Vasoactive agent therapy, } \mathrm{n} \\
\text { (\%) }\end{array}$ & $10(22.7)$ & $12(17.9)$ & 0.533 & $15(32.6)$ & $\begin{array}{l}18 \\
(26.5)\end{array}$ & 0.478 \\
\hline CRRT, n (\%) & $3(6.8)$ & $9(13.4)$ & 0.432 & $7(15.2)$ & $\begin{array}{l}14 \\
(20.6)\end{array}$ & 0.468 \\
\hline CVC, n (\%) & $19(43.2)$ & $26(38.8)$ & 0.646 & $36(78.3)$ & $\begin{array}{l}44 \\
(64.7)\end{array}$ & 0.121 \\
\hline IMV, n (\%) & $34(77.3)$ & $31(46.3)$ & 0.001 & $32(69.6)$ & $\begin{array}{l}25 \\
(36.8)\end{array}$ & 0.001 \\
\hline Length of IMV (days) & $3(2,6)$ & $3(2,10)$ & 0.646 & $3(2,7)$ & $3(2,8)$ & 0.586 \\
\hline $\begin{array}{l}\text { Length of IMV } \geq 3 \text { days, } n \\
(\%)\end{array}$ & $21(61.8)$ & $19(61.3)$ & 0.969 & $22(68.8)$ & $\begin{array}{l}18 \\
(72.0)\end{array}$ & 0.790 \\
\hline Mortality, n (\%) & $21(47.7)$ & $15(22.4)$ & 0.005 & $24(52.2)$ & $\begin{array}{l}17 \\
(25.0)\end{array}$ & 0.003 \\
\hline
\end{tabular}

Data are presented as mean $\pm S D$, median (IQR), or $n(\%)$. P values comparing DVT and non-DVT groups were from a two-sample t-test, Mann-Whitney $U$ test, $\chi^{2}$ test, or Fisher exact test. $P<0.05$ was considered statistically significant.

$\chi^{2}$ test comparing DVT and non-DVT groups.

$\chi^{2}$ test comparing all subcategories.

Abbreviations: APACHE, Acute Physiology and Chronic Health Evaluation; ARDS, acute respiratory disease syndrome; BMI, body mass index; CRRT, continuous renal replacement therapy; CVC, central venous catheterization; DVT, deep venous thrombosis; $\mathrm{FiO}_{2}$, fraction of inspired oxygen; ICU, intensive care unit; IMV, invasive mechanical ventilation; IQR, interquartile range; LMWH, low molecular weight heparin; mild, $200 \mathrm{mmHg} \square \mathrm{PaO}_{2} / \mathrm{FiO}_{2} \leq 300 \mathrm{mmHg}$; moderate, $100 \mathrm{mmHg} \square \mathrm{PaO}{ }_{2} / \mathrm{FiO}_{2} \leq 200 \mathrm{mmHg}$; $\mathrm{PaO}_{2}$, partial pressure of arterial oxygen; $\mathrm{SD}$, standard deviation; severe, $\mathrm{PaO}_{2} / \mathrm{FiO}_{2} \leq 100 \mathrm{mmHg}$; SOFA, Sequential Organ Failure Assessment; VTE, venous thromboembolism.

To determine whether the independent contributors to DVT differed between direct and indirect ARDS, multivariable logistic regression models for DVT were applied in the overall study cohort and then in the 
direct and indirect ARDS groups, respectively (Table 5). In order to reduce data duplication, we did not include thrombus prediction scores and disease severity scores in the multiple regression models.

Because all patients with sedation received IMV, there was a certain degree of overlap between these two variables, so we did not incorporate sedative therapy in the multivariate regression. In the combined and direct ARDS cohorts, age, serum creatinine level, and IMV were independently associated with DVT. In the indirect ARDS group, the independent contributors to DVT were age $(P=0.015)$ and

Table 5

Independent Predictors of Deep Vein Thrombosis in Patients with Direct and Indirect Acute Respiratory Distress Syndrome

\begin{tabular}{|c|c|c|c|c|c|c|c|}
\hline \multirow[t]{2}{*}{ Characteristics } & \multicolumn{2}{|c|}{$\begin{array}{l}\text { Total ARDS (N= } \\
\text { 225) }\end{array}$} & \multicolumn{2}{|c|}{$\begin{array}{l}\text { Direct ARDS }(n= \\
111)\end{array}$} & \multicolumn{2}{|c|}{$\begin{array}{l}\text { Indirect ARDS (n } \\
=114 \text { ) }\end{array}$} & \multirow{2}{*}{$\begin{array}{l}P \text { Value } \\
\text { for } \\
\text { Interaction } \\
\text { With } \\
\text { ARDS } \\
\text { Type }\end{array}$} \\
\hline & $\begin{array}{l}\text { Adjusted } \\
\text { OR (95\% } \\
\text { Cl) }\end{array}$ & $\begin{array}{l}P \\
\text { value }\end{array}$ & $\begin{array}{l}\text { Adjusted } \\
\text { OR (95\% } \\
\text { Cl) }\end{array}$ & $\begin{array}{l}P \\
\text { value }\end{array}$ & $\begin{array}{l}\text { Adjusted } \\
\text { OR } \\
(95 \% \mathrm{Cl})\end{array}$ & $\begin{array}{l}\mathrm{P} \\
\text { value }\end{array}$ & \\
\hline $\begin{array}{l}\text { Age } \\
\text { (per } 10 \text { years) }\end{array}$ & $\begin{array}{l}1.422 \\
(1.147- \\
1.763)\end{array}$ & 0.001 & $\begin{array}{l}1.504 \\
(1.025- \\
2.207)\end{array}$ & 0.037 & $\begin{array}{l}1.410 \\
(1.070- \\
1.856)\end{array}$ & 0.015 & 0.030 \\
\hline $\begin{array}{l}\text { Serum } \\
\text { creatinine (per } \\
10 \mu \mathrm{mol} / \mathrm{L})\end{array}$ & $\begin{array}{l}0.939 \\
(0.908- \\
0.970)\end{array}$ & $<001$ & $\begin{array}{l}0.857 \\
(0.789- \\
0.930)\end{array}$ & $<.001$ & $\begin{array}{l}0.971 \\
(0.936- \\
1.008)\end{array}$ & 0.120 & 0.006 \\
\hline $\begin{array}{l}\text { Procalcitonin } \\
(\mathrm{ng} / \mathrm{mL})\end{array}$ & $\begin{array}{l}1.033 \\
(0.997- \\
1.070)\end{array}$ & 0.071 & $\begin{array}{l}1.035 \\
(0.975- \\
1.099)\end{array}$ & 0.264 & $\begin{array}{l}1.035 \\
(0.989- \\
1.084)\end{array}$ & 0.134 & \\
\hline $\begin{array}{l}\text { D-dimer } \\
(\mu \mathrm{g} / \mathrm{ml})\end{array}$ & $\begin{array}{l}1.065 \\
(0.985- \\
1.151)\end{array}$ & 0.114 & $\begin{array}{l}1.059 \\
(0.852- \\
1.317)\end{array}$ & 0.606 & $\begin{array}{l}1.056 \\
(0.971- \\
1.149)\end{array}$ & 0.205 & \\
\hline $\mathrm{PaO}_{2} / \mathrm{FiO}_{2}$ & $\begin{array}{l}0.996 \\
(0.990- \\
1.002)\end{array}$ & 0.223 & $\begin{array}{l}0.996 \\
(0.986- \\
1.006)\end{array}$ & 0.453 & $\begin{array}{l}0.995 \\
(0.987- \\
1.004)\end{array}$ & 0.295 & \\
\hline IMV & $\begin{array}{l}3.168 \\
(1.579- \\
6.356)\end{array}$ & 0.001 & $\begin{array}{l}5.272 \\
(1.536- \\
18.100)\end{array}$ & 0.008 & $\begin{array}{l}2.787 \\
(1.144- \\
6.792)\end{array}$ & 0.024 & \\
\hline
\end{tabular}

Multivariable logistic regression was performed in the overall ARDS cohort and then in the direct ARDS and indirect ARDS groups separately. The interactions of ARDS type (direct or indirect) with age, serum creatinine level, level of procalcitonin, level of D-dimer, $\mathrm{PaO}_{2} / \mathrm{FiO}_{2}$, and IMV were included in the regression analysis.

Abbreviations: ARDS, acute respiratory disease syndrome; $\mathrm{Cl}$, confidence interval; $\mathrm{DVT}$, deep venous thrombosis; $\mathrm{FiO}_{2}$, fraction of inspired oxygen; IMV, invasive mechanical ventilation; OR, odds ratio; $\mathrm{PaO}_{2}$, partial pressure of arterial oxygen. 
IMV $(P=0.024)$. However, in contrast, the occurrence of DVT increased more significantly with increasing age in those with direct ARDS than in those with indirect ARDS (test for interaction, $P=0.030$; Fig. 2). Distinct from direct ARDS, the serum creatinine level was not independently associated with increased DVT in the indirect ARDS group (test for interaction, $P=0.006$; Fig. 3 ).

We propose three new ways of combining forecasting models for screening for DVT based on the significant risk factors. The sensitivity and specificity of the corresponding receiver operating characteristic curves of the proposed models were not inferior to those of the Padua prediction score and the Caprini score for screening for DVT (Fig. 4A - C).

Kaplan-Meier survival curves showed that patients with DVT had a significantly lower survival rate within 28 days after ARDS than patients without DVT, not only in the overall ARDS cohort but also in the direct and indirect ARDS groups ( $P<0.001$ [Fig. 5A]; $P=0.004$ [Fig. 5B]; and $P=0.007$ [Fig. 5C], respectively).

\section{Discussion}

The main goal of this study was to evaluate the prevalence of, the risk factors for, and the prognosis of DVT in patients with ARDS and to determine whether predictors of DVT differ between those with direct and indirect ARDS. We eventually enrolled 225 patients with ARDS in this study, 111 of whom had direct ARDS and 114, indirect ARDS. The prevalence of DVT on ultrasound scans in the overall group of patients with ARDS was as high as $40.0 \%$, followed by an undifferentiated prevalence between the cohorts with direct and indirect ARDS (39.6\% vs $40.4 \% ; P=0.913$ ). Advanced age, serum creatinine level, and IMV were independently associated with DVT in the overall ARDS group as well as in the direct ARDS cohort. In the indirect ARDS cohort, however, increased DVT was only associated with advanced age and IMV. Patients with DVT had more adverse outcomes than those without

DVT, not only in the overall ARDS cohort but also in the direct and indirect ARDS groups. To our knowledge, this research is the first systematic description of DVT in patients with ARDS and of distinct associations among clinical characteristics and DVT in patients with direct and indirect ARDS.

In 2002, Greets et al. reported that the rates of objectively confirmed DVT in 4 prospective studies ranged from $13-31 \%$ [26]. In recent years, some research showed that, despite the use of guideline-recommended thromboprophylaxis, the incidence of DVT is still as high as $14-37.2 \%$ in critically ill patients [2, 3]. Zhang et al. pointed out that the cumulative incidence of VTE at 7, 14, 21, and 28 days was $4.45 \%, 7.14 \%, 7.53 \%$, and $9.55 \%$, respectively, in patients admitted to ICUs in China, even though the patients received guidelinerecommended thromboprophylaxis [4]. Several factors probably account for the notably higher prevalence of DVT in our patients. First, most of the previously mentioned studies focused on critically ill patients who were in the ICU for other reasons. ARDS is a more serious type of critical illness that shows an overwhelming systemic inflammatory process accompanied by alveolar epithelial and vascular endothelial injury and an abnormal blood coagulation mechanism associated with significant death and may have a higher risk of DVT. Multiple studies have also suggested that the incidence of DVT in patients with ARDS from coronavirus disease 2019 or influenza $A\left(\mathrm{H}_{1} \mathrm{~N}_{1}\right)$ was as high as $42.2-85.4 \%$ [27-30]. 
These conditions indicate that direct ARDS itself may be a risk factor for DVT. As our results show, both direct and indirect ARDS had extremely high incidence rates of DVT. Second, some researchers defined VTE as a pulmonary embolism, proximal DVT, and/or symptomatic distal DVT, thereby excluding asymptomatic isolated distal DVT, which could probably be identified only by screening ultrasound [3]. Furthermore, the heterogeneous patient populations, such as those with different primary conditions, different numbers of days in the hospital, and different preventive measures, may represent a variety incidence.

Advanced age is a well-recognized risk factor for DVT in hospitalized patients, especially in critically ill patients, which has been included in a variety of thrombosis prevention scoring systems [22, 23]. As expected, in this study, the independent association of increased DVT with advanced age was found in both the direct and indirect ARDS cohorts. Interestingly, however, the contribution of advanced age to DVT differed in the different ARDS cohorts. The prevalence of DVT increased more significantly with advancing age in patients with direct ARDS than in those with indirect ARDS. The reason for this phenomenon may be partly, as previous studies have shown $[14,31]$ that, in our study, the patients in the indirect ARDS group also displayed more severe disease (higher APACHE II scores and higher SOFA scores) than those in the direct ARDS group, so the effect of advanced age on the overall condition of indirect ARDS was relatively small.

We found an independent association between serum creatinine levels and DVT in our patients. To our knowledge, however, this study is the first to assess differences in DVT related to renal function in ARDS by direct or indirect etiology. We found that the independent association between the serum creatinine level and the incidence of DVT in ARDS is modified by the underlying ARDS risk factors, with the protective effect on DVT of higher levels of serum creatinine being limited to patients with direct ARDS. However, we did not find a correlation between serum creatinine level and DVT in patients with indirect ARDS, which may be due to the more serious renal impairment and coagulation dysfunction in indirect ARDS, thus weakening the correlation between these two factors. Renal function was associated with dysregulation in coagulation in proportion to the severity of the renal impairment [32]. Some studies have demonstrated that chronic kidney disease and acute kidney injury (AKI) are independent risk factors for VTE [33, 34]. Al-Dorzi et al. pointed out that, for critically ill patients, neither AKI nor end-stage renal disease was an independent risk factor for VTE [35]. McMahon et al. found that AKI increases the risk for hospitalization-related VTE in a large, heterogeneous population that includes medical and surgical patients. However, this relationship was not seen in patients with traumatic injuries [36]. Some studies have shown that LWMH may have different levels of bioaccumulation in the case of renal insufficiency $[37,38]$. The study by Cook et al. indicated that the incidence of DVT for patients with renal insufficiency in ICU who received dalteparin 5,000 IU once daily was 5.1\% [39], which was far lower than that in the overall population of critically ill patients who received preventive treatment recommended by the guidelines [2-4]. So we speculat that the same dose of LWMH may play a stronger role in the prevention of DVT in the case of renal insufficiency. Unfortunately, due to the retrospective nature of the study, the decrease of LWMH metabolism in patients with AKI and higher level of serum creatinine was based on the conjecture of clinical data analysis, and we did not detect the activity of anti-factor Xa. 
ARDS is a clinical syndrome with high mortality manifested by severe acute hypoxemia, which usually requires MV, especially IMV [8]. With IMV, sedation and immobilization are often performed simultaneously, which would aggravate blood stasis and increase the risk of DVT. Some studies have shown that IMV is a high-risk factor for DVT $[28,40]$. Knudso et al. pointed out that IMV administered for more than 3 days is an independent risk factor for VTE [41]. As the duration of IMV increased, the risk of DVT increased [3]. Our research showed that both IMV and sedation were risk factors for DVT. Because all sedated patients in our study were treated with IMV, we only included IMV in the multivariate regression analysis. The results showed that IMV was an independent risk factor for DVT in both direct and indirect ARDS cohorts. However, in our study, compared with patients in the non-DVT group, the duration of IMV in the DVT group did not increase significantly, possibly because our small number of cases resulted in no statistically significant difference.

In direct and indirect ARDS cohorts, neither the APACHE II score nor the SOFA score was associated with the occurrence of DVT, presumably because the serum creatinine level, which was negatively correlated with the occurrence of DVT, was included in these two scoring systems [24, 25], thus weakening the correlation between severity scores and DVT.

Differences in predictors of DVT between direct and indirect ARDS partly support the growing body of literature suggesting that there are subphenotypes of ARDS that affect clinical outcomes [12-14, 16]. Our results suggest that subgroup analyses of ARDS are probably beneficial for stratifying and predicting the risk of DVT. We used age, IMV, and serum creatinine levels to predict DVT in the overall and the direct and indirect ARDS cohorts, respectively, and found that, in ARDS, the combined application of these indicators was not inferior to the current commonly used thrombus prediction scores, such as the Padua prediction score [23] and the Caprini score [22], for screening for DVT. Especially for direct ARDS, the combination of age, IMV, and serum creatinine level yielded a sensitivity of $81.8 \%$ and a specificity of $69.6 \%$ for scanning for DVT. A possible reason is that the Padua prediction score and the Caprini score apply to the general medical and surgical patients in the hospital. As a serious clinical pathophysiological syndrome with an overwhelming inflammatory response and coagulation abnormalities, ARDS has unique clinical characteristics and serious complications. The predictive value of the commonly used thrombus prediction method may be limited to screening for DVT in a patient with a critical illness such as ARDS.

Similar to the results of some previous studies [29,42,43], our results showed that DVT was associated with adverse outcomes in all the ARDS cohorts. Although there was no significant difference between length of stay in the hospital and length of stay in the ICU, Kaplan-Meier curves showed that the 28-day survival rate of patients with DVT was significantly lower than that of patients without DVT in all the ARDS cohorts.

Our study has some limitations. First, our sample size was small, which may underestimate the influence on DVT of factors such as obesity, being bedridden, and the insertion of a central venous catheter.

Second, some patients had ultrasound scans only in the early stage of ARDS and did not have continuous dynamic monitoring, which may cause the incidence of DVT to be underestimated. Third, due 
to the critical condition of patients with ARDS, CTPA examinations were restricted. We performed CTPA examinations on only 3 patients with a high suspicion of PE and then confirmed the diagnosis of $P E$, which significantly underestimated the incidence of PE. Finally, this study is a retrospective study. We hope to conduct a prospective investigation to further clarify the incidence of DVT in patients with different subtypes of ARDS, to determine the corresponding risk factors, and to explore optimized individualized preventive measures in the case of ARDS to reduce DVT-related adverse prognoses.

\section{Conclusions}

The incidence of DVT is extremely high in patients with ARDS and may be associated with adverse outcomes. The risk factors for DVT are age, serum creatinine level, and IMV in ARDS. The combination of risk factors yielded three prediction models for screening for DVT that are superior to the Padua prediction score and the Caprini score in different ARDS subgroups. We suspect that DVT is probably an additional risk factor for death of ARDS in hospitalized patients. The classification and analysis of ARDS may help to provide more accurate screening for DVT and risk stratification and lead to corresponding measures to improve the clinical outcome of patients with ARDS.

\section{Abbreviations}

AKI, acute kidney injury; APACHE, Acute Physiology and Chronic Health Evaluation ARDS = acute respiratory distress syndrome; $\mathrm{AUC}$, area under the curve; $\mathrm{Cl}$, confidence interval; CTPA, computed tomography pulmonary angiography; DVT, deep vein thrombosis; $\mathrm{FiO}_{2}$, fraction of inspired oxygen; ICU, intensive care unit; IMV, invasive mechanical ventilation; IQR, interquartile range; MV, mechanical ventilation; $\mathrm{OR}$, odds ratio; $\mathrm{PaO}_{2}$, partial pressure of arterial oxygen; $\mathrm{PCT}$, procalcitonin; $\mathrm{PE}$, pulmonary embolism; ROC, receiver operating characteristic; SD, standard deviation; SOFA, Sequential Organ Failure Assessment; VTE, venous thromboembolism.

\section{Declarations}

\section{Acknowledgements}

We would like to thank the following doctors for taking part in the diagnosis and treatment of patients with ARDS: Jing Wang, Ling Wang, Jun Zhang and Song Mi from the Department of Pulmonary and Critical Care Medicine, Beijing Chao-Yang Hospital, Capital Medical University, Beijing, People's Republic China.

\section{Authors' contributions}

N.C., C.J., and H.C. designed the study, collected clinical data, analyzed the data, and wrote the manuscript. N.C., C.J., and H.C. contributed equally and shared first authorship. Y. G. and L.Y. collected clinical data. X.F. and L.Z. helped manage the research, performed the statistical analyses, and revised 
the paper. X.F. and L.Z. contributed equally to this article and share corresponding authorship. All authors read and approved the final manuscript.

Funding This work was supported by the Research on prevention and control of major chronic noncommunicable diseases of China (Grant NO. 2016YFC1304603 to Dr Xiaokai Feng).

\section{Availability of data and materials}

All data analyzed during the study are presented in the main manuscript. The anonymous dataset is available from the corresponding author upon reasonable request.

\section{Ethics and approval and consent to participate}

This retrospective study involving human participants was approved by the ethics committee of the Beijing Chao-Yang Hospital, Capital Medical University (2020-ke-429) and the First Affiliated Hospital of Shandong First Medical University (S003) and was conducted in accordance with the 1964 Helsinki Declaration and its later amendments or comparable ethical standards.

\section{Consent for publication}

Not applicable.

\section{Competing interests}

The authors declare that they have no competing interests.

\section{Author details}

1 Department of Pulmonary and Critical Care Medicine, Beijing Chao-Yang Hospital, Capital Medical University, Beijing, 100020, People's Republic China; ${ }^{2}$ Beijing Institute of Respiratory Medicine, Beijing 100020, People's Republic China; ${ }^{3}$ Department of Intensive Care Unit, Shandong Qianfo Mountain hospital, the First Affiliated Hospital of Shandong First Medical University Na Cui, Chunguo Jiang, and Hairong Chen contributed equally.

\section{References}

1. Di Nisio M, van Es N, Büller HR. Deep vein thrombosis and pulmonary Lancet. 2016;388(10063):3060-3073.

2. Gibson CD, Colvin MO, Park MJ, et al. Prevalence and predictors of deep vein thrombosis in critically ill medical patients who underwent diagnostic duplex ultrasonography. J Intensive Care Med. 2020;35(10):1062-1066. 
3. Kaplan D, Casper TC, Elliott CG, et al. VTE incidence and risk factors in patients with severe sepsis and septic shock. Chest. 2015;148(5):1224-1230.

4. Zhang $\mathrm{C}$, Zhang Z, Mi J, et al. The cumulative venous thromboembolism incidence and risk factors in intensive care patients receiving the guideline-recommended thromboprophylaxis. Medicine (Baltimore) 2019;98(23):e15833.

5. Chang JC. Acute respiratory distress syndrome as an organ phenotype of vascular microthrombotic disease: Based on hemostatic theory and endothelial molecular pathogenesis. Clin Appl Thromb Hemost. 2019;25:1076029619887437.

6. Gando S, Kameue T, Matsuda N, Sawamura A, Hayakawa M, Kato H. Systemic inflammation and disseminated intravascular coagulation in early stage of ALI and ARDS: Role of neutrophil and endothelial activation. Inflammation. 2004;28(4):237-244.

7. Fan E, Brodie D, Slutsky AS. Acute respiratory distress syndrome: Advances in diagnosis and treatment. JAMA. 2018;319(7):698-710.

8. ARDS Definition Task Force, Ranieri VM, Rubenfeld GD, et al. Acute respiratory distress syndrome: The Berlin definition. JAMA. 2012;307(23):2526-2533.

9. Bellani G, Laffey JG, Pham T, et al. Epidemiology, patterns of care, and mortality for patients with acute respiratory distress syndrome in intensive care units in 50 countries. JAMA. 2016;315(8):788800.

10. Eworuke E, Major JM, Gilbert McClain LI. National incidence rates for acute respiratory distress syndrome (ARDS) and ARDS cause-specific factors in the United States (2006- 2014). J Crit Care. 2018;47:192-197.

11. Li S, Zhao D, Cui J, Wang L, Ma X, Li Y. Prevalence, potential risk factors and mortality rates of acute respiratory distress syndrome in Chinese patients with sepsis. J Int Med Res. 2020;48(2):300060519895659.

12. Shaver $\mathrm{CM}$, Bastarache JA. Clinical and biological heterogeneity in acute respiratory distress syndrome: Direct versus indirect lung injury. Clin Chest Med. 2014;35(4):639- 653.

13. Anan K, Kawamura K, Suga M, Ichikado K. Clinical differences between pulmonary and extrapulmonary acute respiratory distress syndrome: A retrospective cohort study of prospectively collected data in Japan. J Thorac Dis. 2018;10(10):5796-5803.

14. Luo L, Shaver CM, Zhao Z, et al. Clinical predictors of hospital mortality differ between direct and indirect ARDS. Chest 2017;151(4):755-763.

15. Morisawa K, Fujitani S, Taira Y, et al. Difference in pulmonary permeability between indirect and direct acute respiratory distress syndrome assessed by the transpulmonary thermodilution technique: A prospective, observational, multi-institutional study. J Intensive Care. 2014;2(1):24.

16. Calfee CS, Janz DR, Bernard GR, et al. Distinct molecular phenotypes of direct vs indirect ARDS in single-center and multicenter studies. Chest. 2015;147(6):1539-1548.

17. Perl M, Lomas-Neira J, Venet F, Chung CS, Ayala A. Pathogenesis of indirect (secondary) acute lung injury. Expert Rev Respir Med. 2011;5(1):115-126. 
18. Gong MN, Wei Z, Xu LL, Miller DP, Thompson BT, Christiani DC. Polymorphism in the surfactant protein-B gene, gender, and the risk of direct pulmonary injury and ARDS. Chest. 2004;125(1):203211.

19. Pelosi P, D'Onofrio D, Chiumello D, et al. Pulmonary and extrapulmonary acute respiratory distress syndrome are different. Eur Respir J Suppl. 2003;42:48s-56s.

20. Rudski LG, Lai WW, Afilalo J, et al. Guidelines for the echocardiographic assessment of the right heart in adults: A report from the American Society of Echocardiography endorsed by the European Association of Echocardiography, a registered branch of the European Society of Cardiology, and the Canadian Society of Echocardiography. J Am Soc Echocardiogr. 2010;23(7):685-713; quiz 786-688.

21. Singer M, Deutschman CS, Seymour CW, et al. The Third International Consensus Definitions for Sepsis and Septic Shock (Sepsis-3). JAMA. 2016;315(8):801-810.

22. Cronin M, Dengler N, Krauss ES, et al. Completion of the updated Caprini risk assessment model (2013 Version). Clin Appl Thromb Hemost. 2019;25:1076029619838052.

23. Barbar S, Noventa F, Rossetto V, et al. A risk assessment model for the identification of hospitalized medical patients at risk for venous thromboembolism: The Padua Prediction Score. J Thromb Haemost. 2010;8(11):2450-2457.

24. Knaus WA, Draper EA, Wagner DP, Zimmerman JE. APACHE II: A severity of disease classification system. Crit Care Med. 1985;13(10):818-829.

25. Lambden S, Laterre PF, Levy MM, Francois B. The SOFA score-development, utility and challenges of accurate assessment in clinical trials. Crit Care. 2019;23(1):374.

26. Geerts W, Cook D, Selby R, Etchells E. Venous thromboembolism and its prevention in critical care. J Crit Care. 2002;17(2):95-104.

27. Chen S, Zhang D, Zheng T, Yu Y, Jiang J. DVT incidence and risk factors in critically ill patients with COVID-19. J Thromb Thrombolysis. 2021;51(1):33-39.

28. Ren B, Yan F, Deng Z, et al. Extremely high incidence of lower extremity deep venous thrombosis in 48 patients with severe COVID-19 in Wuhan. Circulation. 2020;142(2):181-183.

29. Zhang L, Feng $X$, Zhang D, et al. Deep vein thrombosis in hospitalized patients with COVID-19 in Wuhan, China: Prevalence, risk factors, and outcome. Circulation. 2020;142(2):114-128.

30. Obi AT, Tignanelli CJ, Jacobs BN, et al. Empirical systemic anticoagulation is associated with decreased venous thromboembolism in critically ill influenza A H1N1 acute respiratory distress syndrome patients. J Vasc Surg Venous Lymphat Disord. 2019;7(3):317-324.

31. Sevransky JE, Martin GS, Mendez-Tellez P, et Pulmonary vs nonpulmonary sepsis and mortality in acute lung injury. Chest. 2008;134(3):534-538.

32. Dobrowolski C, Clark EG, Sood MM. Venous thromboembolism in chronic kidney disease: epidemiology, the role of proteinuria, CKD severity and therapeutics. J Thromb Thrombolysis. 2017;43(2):241-247. 
33. Kuo TH, Li HY, Lin SH. Acute kidney injury and risk of deep vein thrombosis and pulmonary embolism in Taiwan: A nationwide retrospective cohort study. Thromb Res. 2017;151:29-35.

34. Christiansen CF, Schmidt M, Lamberg AL, et al. Kidney disease and risk of venous thromboembolism: a nationwide population-based case-control study. J Thromb Haemost. 2014;12(9):1449-1454.

35. Al-Dorzi HM, Al-Heijan A, Tamim HM, Al-Ghamdi G, Arabi YM. Renal failure as a risk factor for venous thromboembolism in critically III patients: A cohort study. Thromb Res. 2013;132(6):671-675.

36. McMahon MMJ, Collen CJF, Chung CKK, et al. Acute kidney injury during hospitalization increases the risk of VTE. Chest. 2021;159(2):772-780.

37. Mahé I, Aghassarian M, Drouet L, et al. Tinzaparin and enoxaparin given at prophylactic dose For eight days in medical elderly patients with impaired renal function: a comparative pharmacokinetic study. Thromb Haemost. 2007; 97(4):581-586.

38. Schmid P, Brodmann D, Odermatt Y, Fischer AG, Wuillemin WA. Study of bioaccumulation of dalteparin at a therapeutic dose in patients with renal $J$ Thromb Haemost. 2009;7(10):1629-1632.

39. Cook D, Douketis J, Meade M, et al. Venous thromboembolism and bleeding in critically ill patients with severe renal insufficiency receiving dalteparin prophylaxis: Prevalence, incidence and risk factors. Critical Care. 2008;12(2):R32.

40. Rali P, O'Corragain O, Oresanya L, et al. Incidence of venous thromboembolism in coronavirus disease 2019: An experience from a single large academic center. J Vasc Surg Venous Lymphat Disord. 2020; Oct 5:S2213-333X(20)30524-2.

41. Knudson MM, Ikossi DG, Khaw L, Morabito D, Speetzen LS. Thromboembolism after trauma: An analysis of 1602 episodes from the American College of Surgeons National Trauma Data Bank. Ann Surg. 2004;240(3):490-496; discussion 496-498.

42. Malato A, Dentali F, Siragusa S, et al. The impact of deep vein thrombosis in critically ill patients: A meta-analysis of major clinical outcomes. Blood Transfus. 2015;13(4):559- 568.

43. Zerwes S, Hernandez Cancino F, Liebetrau D, et al. [Increased risk of deep vein thrombosis in intensive care unit patients with CoViD-19 infections?-Preliminary data]. [Article in German]. Chirurg. 2020;91(7):588-594.

\section{Figures}




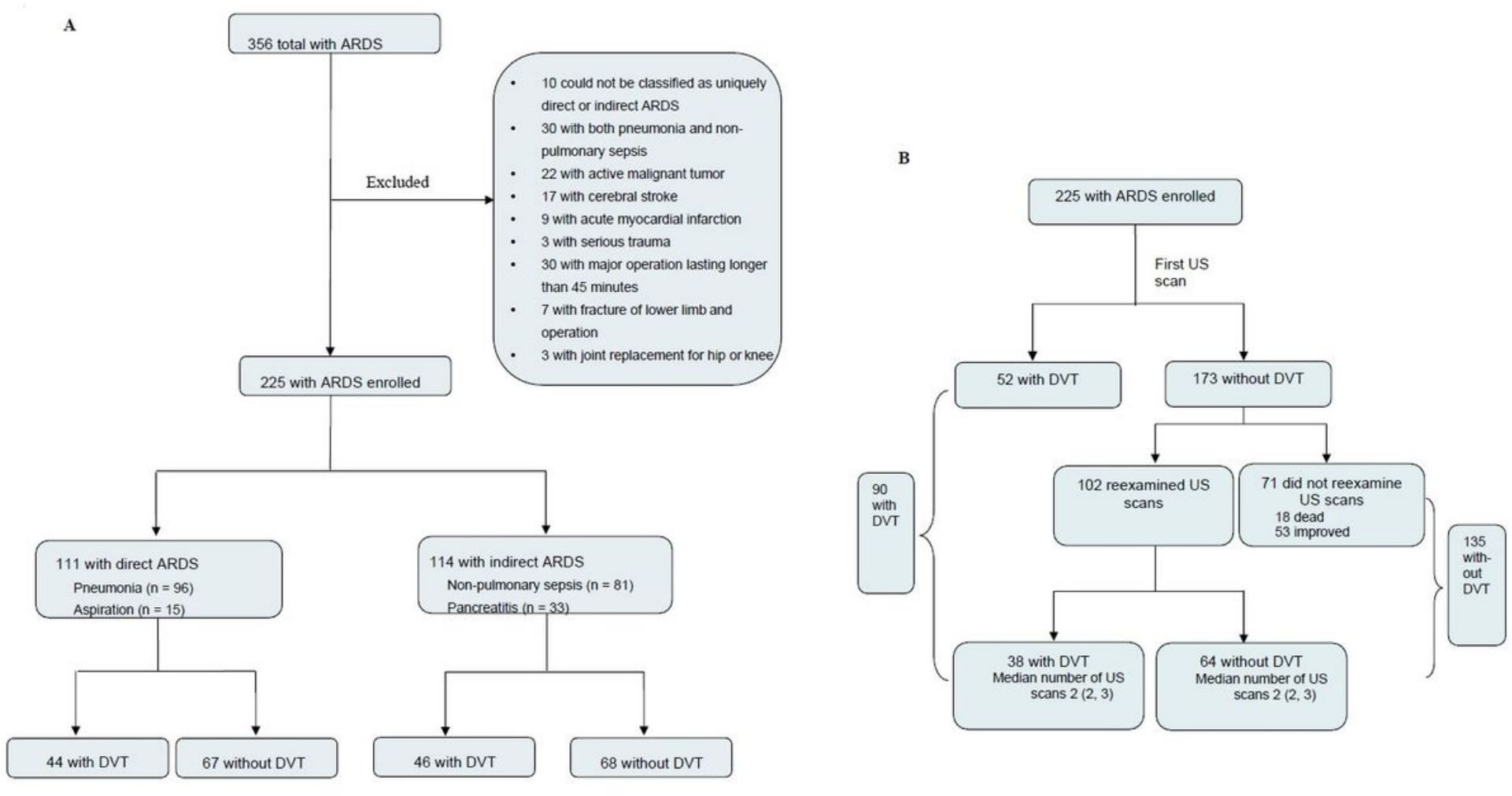

Figure 1

A, B, Study flow chart. A, flow chart for including patients; B, flow chart for screening for DVT. The interval from the diagnosis of ARDS to the occurrence of DVT in the DVT group was $5(2,9)$ days, and the interval from the diagnosis of ARDS to the last ultrasound examination in the non-DVT group was $5(2,11)$ days. There were no differences between the two groups $(P=0.784)$. Abbreviations: ARDS, acute respiratory disease syndrome; DVT, deep vein thrombosis; US, ultrasound. 


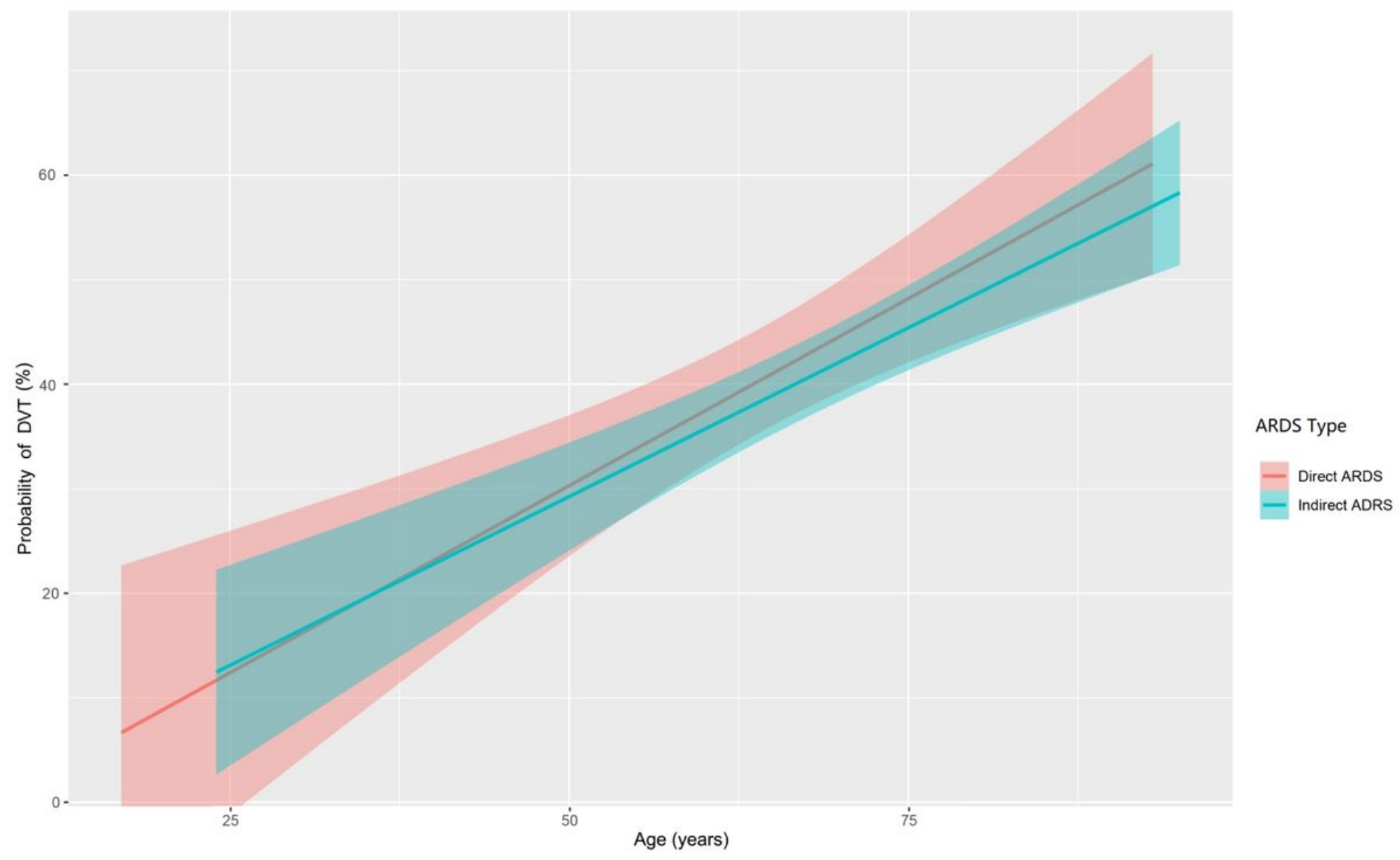

\section{Figure 2}

Prevalence of DVT increased with age in patients with ARDS. The prevalence of DVT increased with age in patients with both direct (red line) and indirect ARDS (blue line). However, the occurrence of DVT increased more significantly with increasing age in the direct ARDS than in the indirect ARDS group (test for interaction; $P=0.030$ ). Data are adjusted for level of serum creatinine, level of procalcitonin, level of $D$ dimer, $\mathrm{PaO} 2 / \mathrm{FiO2}$, and invasive mechanical ventilation. Abbreviations: ARDS, acute respiratory disease syndrome; DVT, deep vein thrombosis; FiO2, fraction of inspired oxygen; $\mathrm{PaO2}$, partial pressure of arterial oxygen. 


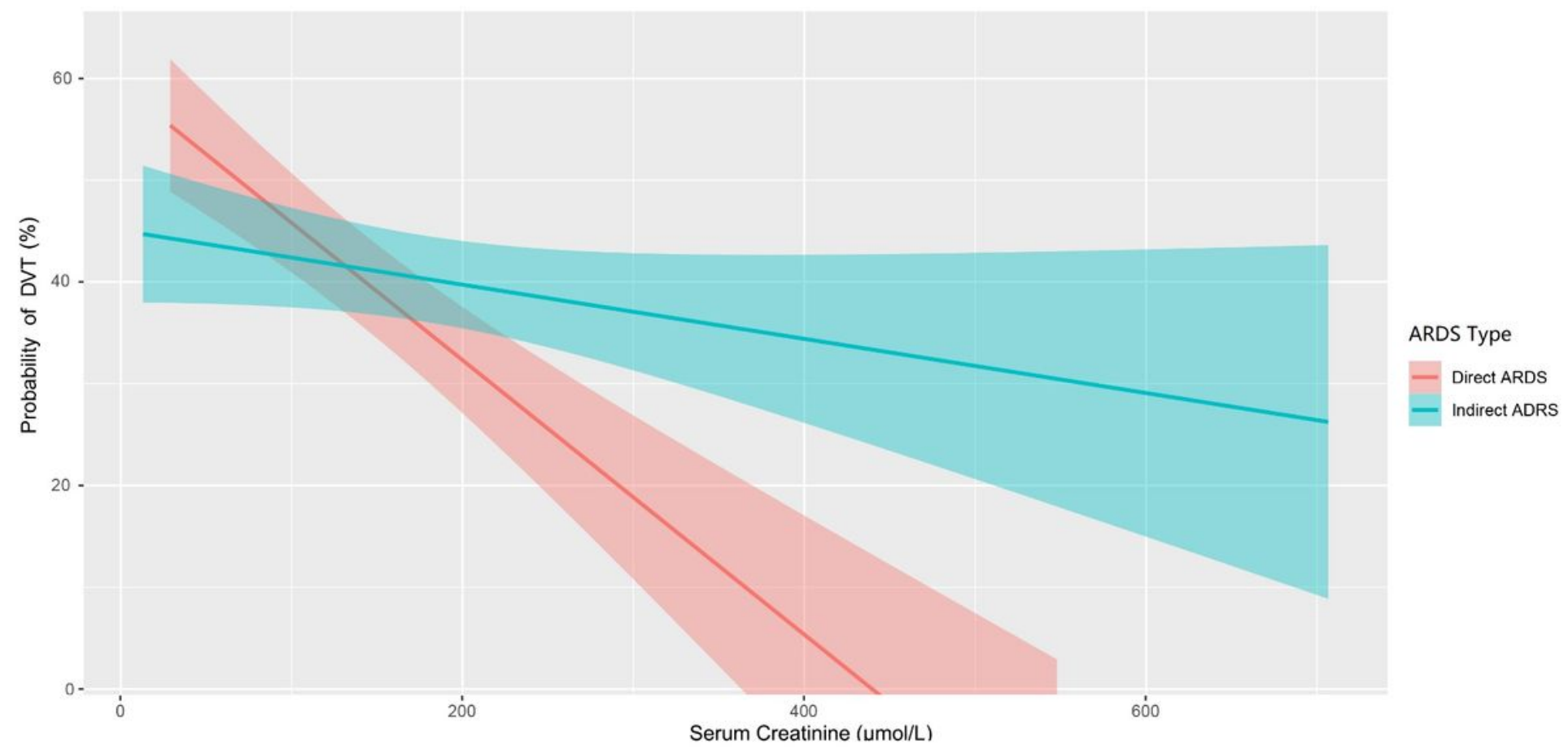

\section{Figure 3}

Prevalence of DVT decreased with serum creatinine levels only in the direct ARDS group. The occurrence of DVT in the direct ARDS group (red line) decreased with increasing serum creatinine levels, whereas serum creatinine levels had no association with DVT in the indirect ARDS group (blue line; test for interaction, $\mathrm{P}=0.006$ ). Data are adjusted for age, procalcitonin levels, $\mathrm{D}$-dimer levels, $\mathrm{PaO} 2 / \mathrm{FiO2}$, and invasive mechanical ventilation. Abbreviations: ARDS, acute respiratory disease syndrome; DVT, deep vein thrombosis; $\mathrm{FiO} 2$, fraction of inspired oxygen; $\mathrm{PaO} 2$, partial pressure of arterial oxygen. 

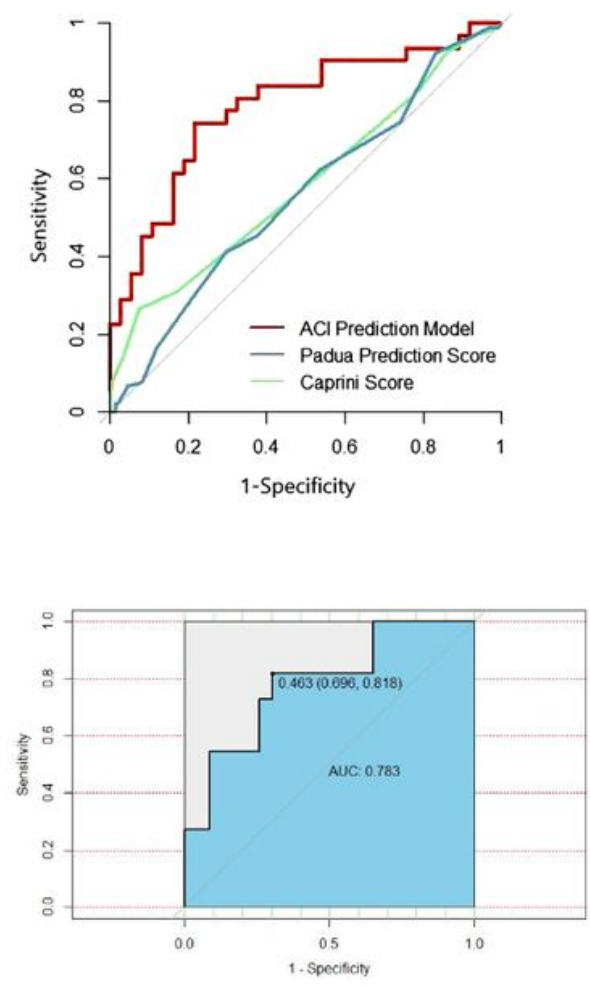
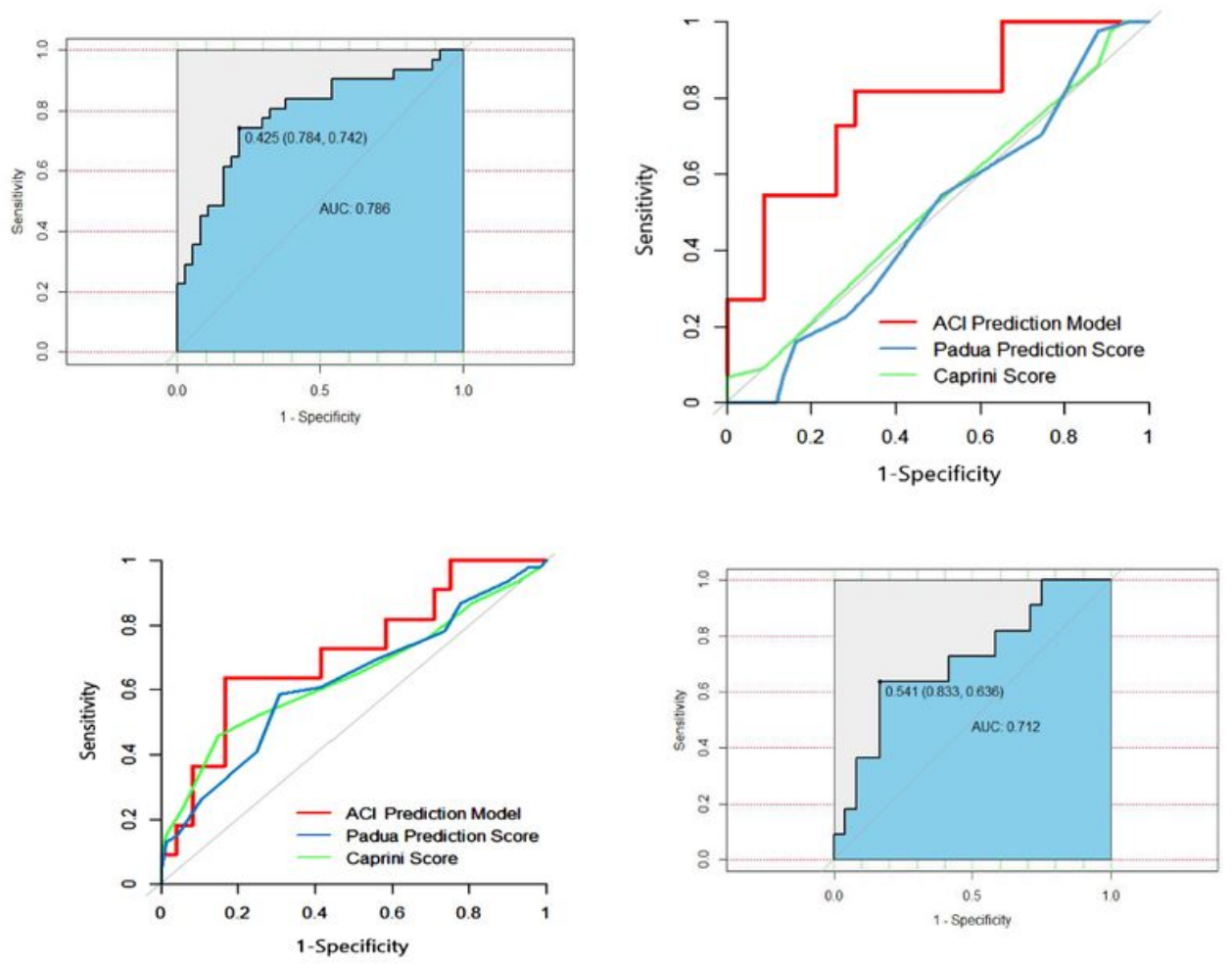

\section{Figure 4}

A-C, Comparison of diagnostic accuracy for screening for DVT of different ROCs in three ARDS cohorts. Patients were split by generating random numbers to produce a training data set $(n * 0.7)$ and a validation data set $(n \star 0.3)$ in the overall, direct, and indirect groups respectively. A, the ACl model which including age, serum creatinine level, and IMV shows satisfactory forecasting ability for DVT (AUC $=0.786 ; 95 \% \mathrm{Cl}$ : 0.673 - 0.898; sensitivity: 74.2\%; specificity: 78.4\%; $P<0.001$ ) significantly higher than that of the Padua prediction score (AUC=0.587; $\mathrm{P}=0.005$ for these two curves) and the Caprini score (AUC $=0.558 ; \mathrm{P}=$ 0.001 for these two curves). $\mathrm{B}$, the $\mathrm{ACl}$ model shows a satisfactory ability to predicting DVT (AUC $=0.783$; $95 \% \mathrm{Cl}: 0.612-0.953$; sensitivity: $81.8 \%$; specificity: $69.6 \% ; \mathrm{P}=0.004)$ significantly surpassed the Padua prediction score (AUC $=0.521 ; \mathrm{P}=0.001$ for these two curves) and the Caprini score (AUC $=0.492 ; \mathrm{P}=$ 0.006 for these two curves). $C$, the ACl model shows satisfactory ability for predicting DVT (AUC $=0.712$; $95 \% \mathrm{Cl}$ : $0.519-0.905$; sensitivity: $63.6 \%$; specificity: $83.3 \% ; \mathrm{P}=0.024)$ has no obvious difference compared with the Padua prediction score (AUC $=0.644 ; \mathrm{P}=0.551$ for these two curves) and the Caprini score (AUC = 0.627; $P=451$ for these two curves). Abbreviations: $A C l=$ age + creatinine + IMV; ARDS, acute respiratory disease syndrome; AUC, area under the curve; $\mathrm{Cl}$, confidence interval; DVT, deep vein thrombosis; IMV, invasive mechanical ventilation; ROC, receiver operating characteristic. 

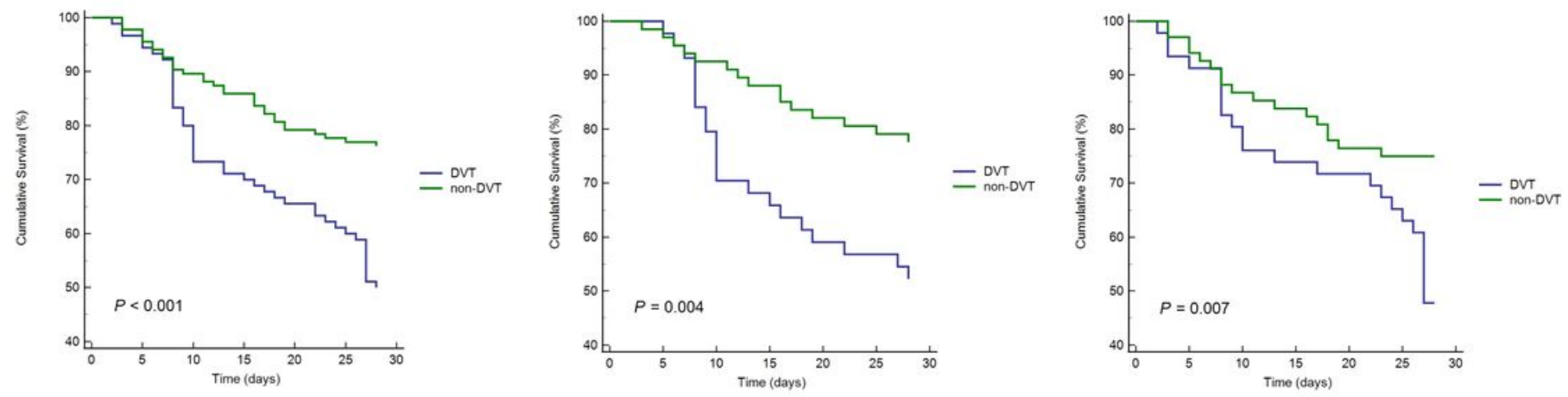

\section{Figure 5}

A-C, Survival curves for patients with and without DVT in the different ARDS cohorts (log-rank test). A, The 28-day survival for patients with and without DVT in the overall ARDS cohort $(P<0.001)$; $B$, the 28-day survival for patients with and without DVT in the direct ARDS cohort $(P=0.004) ; C$, the 28- day survival for patients with and without DVT in the indirect ARDS cohort $(P=0.007)$. Abbreviations: ARDS, acute respiratory disease syndrome; DVT, deep vein thrombosis.

\section{Supplementary Files}

This is a list of supplementary files associated with this preprint. Click to download.

- SupplementalfileillustrationofROCcurves.docx 